\title{
NOMBRE DE CAS DE DIABÈTE DE TYPE 1 ET 2 DIAGNOSTIQUÉS À AMAPÁ ENTRE 2007 ET 2012
}

\section{ARTICLE ORIGINAL}

SOUZA, Kauê de Melo', FACCO, Lucas², FECURY, Amanda Alves³, ARAÚJO, Maria Helena Mendonça de ${ }^{4}$, OLIVEIRA, Euzébio de ${ }^{5}$, DENDASCK, Carla Viana ${ }^{6}$, SOUZA, Keulle Oliveira da ${ }^{7}$, DIAS, Claudio Alberto Gellis de Mattos ${ }^{8}$

SOUZA, Kauê de Melo. Et al. Nombre de cas de diabète de type 1 et 2 diagnostiqués à Amapá entre 2007 et 2012. Revista Científica Multidisciplinar Núcleo do Conhecimento. An 05, Ed. 12, Vol. 01, pp. 18-26. décembre 2020. ISSN: 2448-0959, Lien d'accès: https://www.nucleodoconhecimento.com.br/sante/cas-dediabete, DOI: 10.32749/nucleodoconhecimento.com.br/sante/cas-de-diabete

\section{RÉSUMÉ}

Diabète sucré est une succession de différents types de troubles dans le métabolisme qui se caractérisent par la cause d'un taux élevé de sucre dans le sang. Parce qu'il s'agit d'une maladie avec des facteurs génétiques de type 1 diabète a comme principal facteur de risque hérédité, tandis que le diabète de type 2 en plus de ces facteurs, comprend l'obésité, l'hypertension artérielle, une mauvaise éducation alimentaire et l'âge avancé. Cette étude vise à montrer le nombre de cas de diabète de type 1 et 2 diagnostiqués en Amapá avec les variables sexe, groupe

\footnotetext{
${ }^{1}$ Technicien minier, diplômé de I'Institut fédéral d'Amapá (IFAP).

2 Étudiant du cours de médecine de l'Université fédérale d'Amapá (UNIFAP).

${ }^{3}$ Biomédical, Docteur en maladies tropicales, Professeur et chercheur du Cours médical de l'Université fédérale d'Amapá (UNIFAP).

${ }^{4}$ Médecin, professeur et chercheur du cours de médecine de l'Université fédérale d'Amapá (UNIFAP).

${ }^{5}$ Biologiste, PhD en maladies topiques, professeur et chercheur du cours d'éducation physique de l'Université fédérale du Pará (UFPA).

${ }^{6}$ Théologien, Docteur en psychanalyse, chercheur au Centre de recherche et d'études avancées - CEPA.

${ }^{7}$ Sociologue, étudiant à la maîtrise en études anthropiques en Amazonie, membre du groupe de recherche "Laboratoire d'éducation, d'environnement et de santé " (LEMAS/UFPA).

${ }^{8}$ Biologiste, $\mathrm{PhD}$ en théorie et recherche comportementale, professeur et chercheur du Programme d'études supérieures en éducation professionnelle et technologique (PROFEPT), Institut fédéral d'Amapá (IFAP).
}

RC: 66737

Disponible en: https://www.nucleodoconhecimento.com.br/sante/cas-de-diabete 
d'âge, mode de vie sédentaire, surpoids, tabagisme, entre 2007 et 2012. Les données de la recherche ont été prises auprès du département informatique de SUS, DATASUS (http://datasus.saude.gov.br). Les diabètes sucrés de type 1 et 2 (DM1 et DM2) sont des maladies liées à des perturbations de la production ou à l'utilisation efficace de l'insuline. Le tabagisme, ainsi que le mode de vie sédentaire et l'embonpoint sont des facteurs de risque importants pour le développement du DM2. Le diabète sucré de type 2 fournit le développement de diverses lésions nerveuses organiques. En outre, le DM2, par sa chronicité, permet le développement des rétinopathies, des néphropathies et d'autres conditions négatives à la santé de l'individu.

Mots-clés: Amapá, Diabète sucré, surpoids, mode de vie sédentaire, tabagisme.

\section{INTRODUCTION}

Le diabète sucré est une succession de différents types de troubles du métabolisme qui se caractérisent par un taux élevé de sucre dans le sang, généralement lié à l'absence d'insuline ou d'insuffisance dans l'absorption de la même par le corps (ADA, 2014).

Le diabète de type 1 (DM1) se produit lorsque le corps attaque les cellules responsables de la production d'insuline conduisant à une carence complète de cette hormone dans le corps. II se produit moins souvent parce qu'il est généralement lié à des facteurs génétiques. Le diabète de type 2 (DM2) se produit lorsque le corps ne peut pas absorber l'insuline produite dans le corps en raison de l'incapacité du pancréas à le produire en quantité suffisante. Cela se produit habituellement lorsque l'individu a des antécédents de mauvaise alimentation et de mode de vie sédentaire et qu'il est plus facilement acquis s'il a une tendance héréditaire à la maladie (BRASIL, 2006; MORA et al., 2015).

Les symptômes du diabète de type 1 et 2 comprennent l'augmentation du volume urinaire, l'excès de tête, l'augmentation de la faim, la perte de poids, la fatigue, les

$\mathrm{RC}: 66737$

Disponible en: https://www.nucleodoconhecimento.com.br/sante/cas-de-diabete 
sautes d'humeur, l'hypoglycémie et l'hyperglycémie. Le diabète de type 2 peut ne pas présenter de symptômes avant plusieurs années, l'augmentation de la carence en insuline étant le fusible de l'évolution de ce type (UFRGS, 2016; NOGUEIRA et al., 2015).

Parce qu'il s'agit d'une maladie avec des facteurs génétiques de type 1 diabète a comme principal facteur de risque hérédité, tandis que le diabète de type 2 en plus de ces facteurs, comprend l'obésité, l'hypertension artérielle, une mauvaise éducation alimentaire et l'âge avancé (LIMA et al.; 2014)

La prévention du diabète de type 2 est directement liée à une vie avec de bonnes habitudes alimentaires et des exercices physiques réguliers. Parce qu'il est héréditaire et auto-immune, ce n'est pas la façon de prévenir le diabète de type 1 (MAGALHÃES et al., 2017).

Puisque DM1 est caractérisé par l'insuffisance complète d'insuline, la thérapie d'insuline s'est montrée pour être le meilleur traitement. DM2 parce que c'est une incapacité du corps à produire ou absorber assez d'insuline, le traitement est consacré au maintien du contrôle glycémique du corps, qui inclut des médicaments oraux et des exercices physiques avec une alimentation équilibrée (UFRGS, 2016; SANTOS; FREITAS; PINTO, 2014).

En 2012, le nombre de cas mondiaux de diabète était d'environ 200 millions, tandis que le nombre de cas au Brésil était proche de 10 millions (BRASIL, 2012).

\section{BUT}

Afficher le nombre de cas de diabète de type 1 et 2 diagnostiqués en Amapá avec les variables sexe, groupe d'âge, mode de vie sédentaire, surpoids, tabagisme, entre 2007 et 2012.

RC: 66737

Disponible en: https://www.nucleodoconhecimento.com.br/sante/cas-de-diabete 


\section{MÉTHODE}

Données issues du service informatique de SUS, DATASUS (http://datasus.saude.gov.br), en suivant les étapes : d'abord, l'onglet « Accès à l'information » a été sélectionné, suivi de l'option « Informations de santé (TABNET) » juste après la sous-option « épidémiologie et morbidité », puis le groupe d'options « Hypertension et diabète (HYPERDIA) » a été consulté. Ensuite, l'icône «Hyperdia Enregistrement et suivi des patients hypertendus et diabétiques - depuis 2002» a été sélectionnée, l'onglet «sélectionnez l'option ou cliquez sur la carte» a été ouvert et l'option «Amapá» a été sélectionnée. - si dans la ligne champ l'option « sexe », dans le champ colonne l'option « non actif », et dans le champ contenu l'option « diabète de type 2 », puis dans «périodes disponibles » les données ont été collectées de 2007 à 2012 ; étant la même période utilisée pour toutes les autres collections. L'option « groupe d'âge » a été sélectionnée dans le champ de ligne, dans le champ de colonne l'option « non actif » et dans le champ de contenu l'option « diabète de type 2 ». L'option « sexe » a été sélectionnée dans le champ de ligne, dans le champ de colonne l'option « non actif », et dans le champ de contenu l'option « diabète de type 1 ». L'option groupe d'âge a été sélectionnée dans le champ de ligne, dans le champ de colonne l'option « non actif » et dans le champ de contenu l'option « diabète de type 1 ». L'option « année » a été sélectionnée dans le champ de ligne, dans le champ de colonne l'option « non actif », et dans le champ de contenu l'option « diabète de type 1 ». L'option « année » a été sélectionnée dans le champ de ligne, dans le champ de colonne l'option « non actif », et dans le champ de contenu l'option « diabète de type 2 ». L'option « tabagisme » a été sélectionnée dans le champ de ligne, dans le champ de colonne l'option « non actif » et dans le champ de contenu l'option « diabète de type 1 ». L'option «tabagisme » a été sélectionnée dans le champ de ligne, dans le champ de colonne l'option « non actif », et dans le champ de contenu l'option « diabète de type 2. La compilation des données a été effectuée dans I'application Excel, un composant de Microsoft Office. paquet Corporation. La recherche bibliographique a été effectuée sur des articles scientifiques, à l'aide d'ordinateurs du laboratoire informatique de l'Institut fédéral de l'éducation, des

RC: 66737

Disponible en: https://www.nucleodoconhecimento.com.br/sante/cas-de-diabete 
sciences et de la technologie d'Amapá, Campus Macapá, situé à : Rodovia BR 210 KM 3, s/n - Bairro Brasil Novo , CEP : 68.909-398, Macapá, Amapá, Brésil.

\section{RÉSULTATS}

La figure 1 montre le nombre de cas confirmés de diabète dans l'État d'Amapá entre 2007 et 2012. Plus de cas de diabète de type 2 que de type 1 ont été diagnostiqués.

La figure 1 montre le nombre de cas confirmés de diabète dans l'état d'Amapá entre 2007 et 2012.

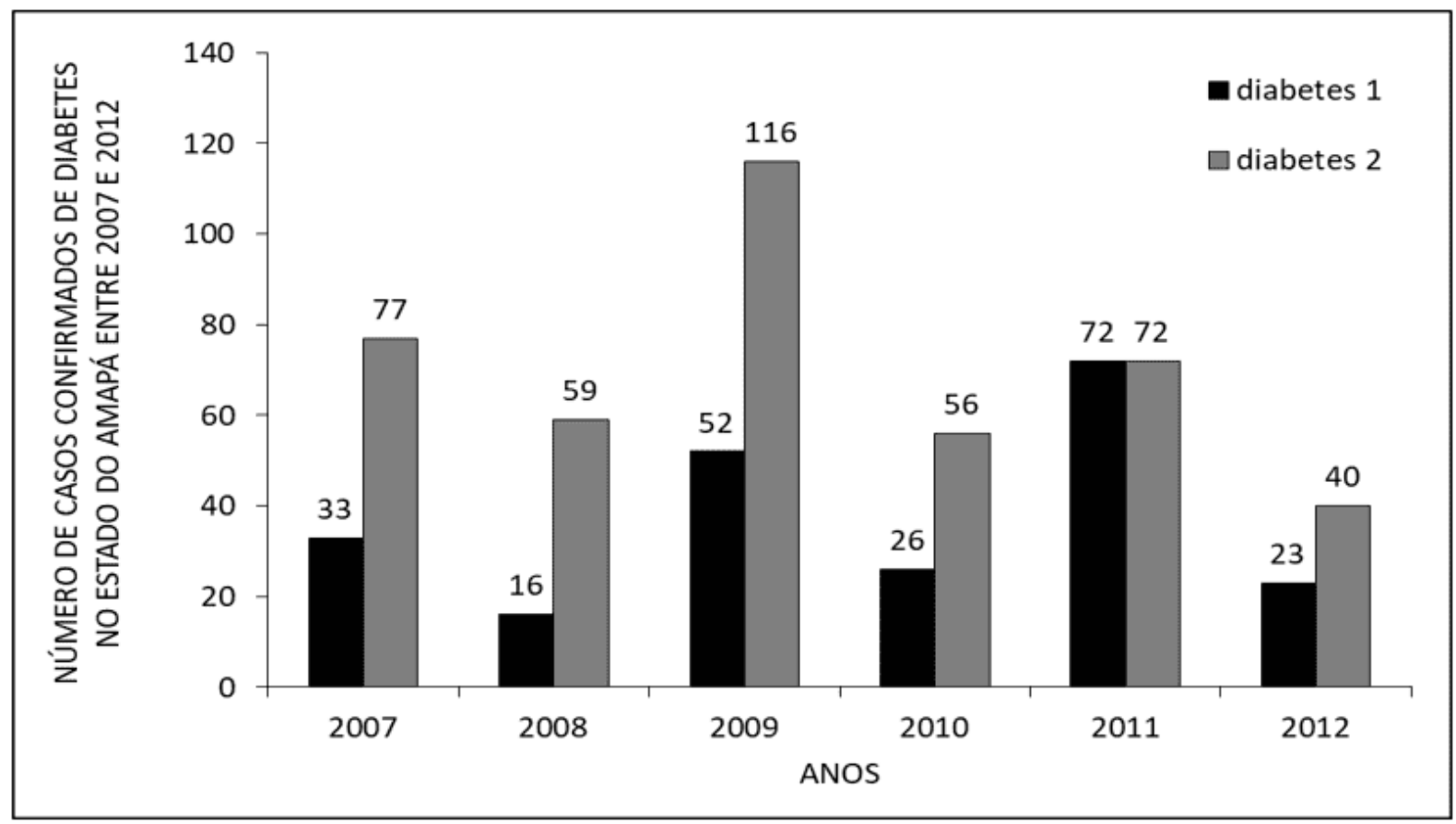

La figure 2 montre le nombre de cas confirmés de diabète de type 1 et 2 dans l'État d'Amapá entre 2007 et 2012 selon le sexe. Le plus grand nombre de diagnostics de diabète de type 1 et 2 était chez les femmes.

RC: 66737

Disponible en: https://www.nucleodoconhecimento.com.br/sante/cas-de-diabete 
La figure 2 montre le nombre de cas confirmés de diabète de type 1 et 2 dans l'État d'Amapá entre 2007 et 2012 selon le sexe.

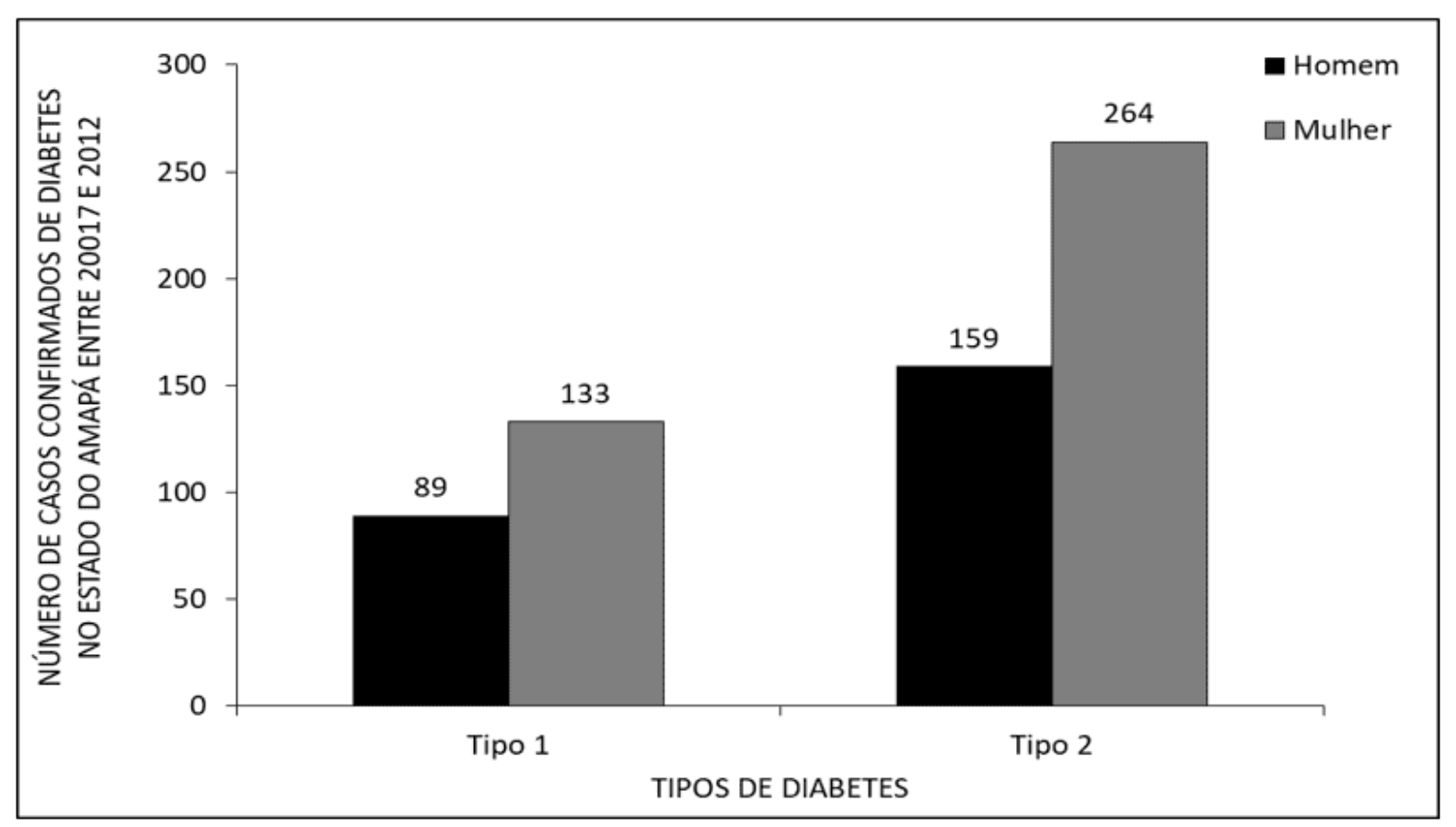

La figure 3 montre le nombre de cas confirmés de diabète de type 1 et 2 dans l'État d'Amapá entre 2007 et 2012 selon le groupe d'âge. Le plus grand nombre de diagnostics de diabète de type 1 et 2 était celui des personnes âgées de 30 à 59 ans, tandis que le plus bas était celui des personnes jusqu'à l'âge de 29 ans.

RC: 66737

Disponible en: https://www.nucleodoconhecimento.com.br/sante/cas-de-diabete 
La figure 3 montre le nombre de cas confirmés de diabète de type 1 et 2 dans l'État d'Amapá entre 2007 et 2012 selon le groupe d'âge.

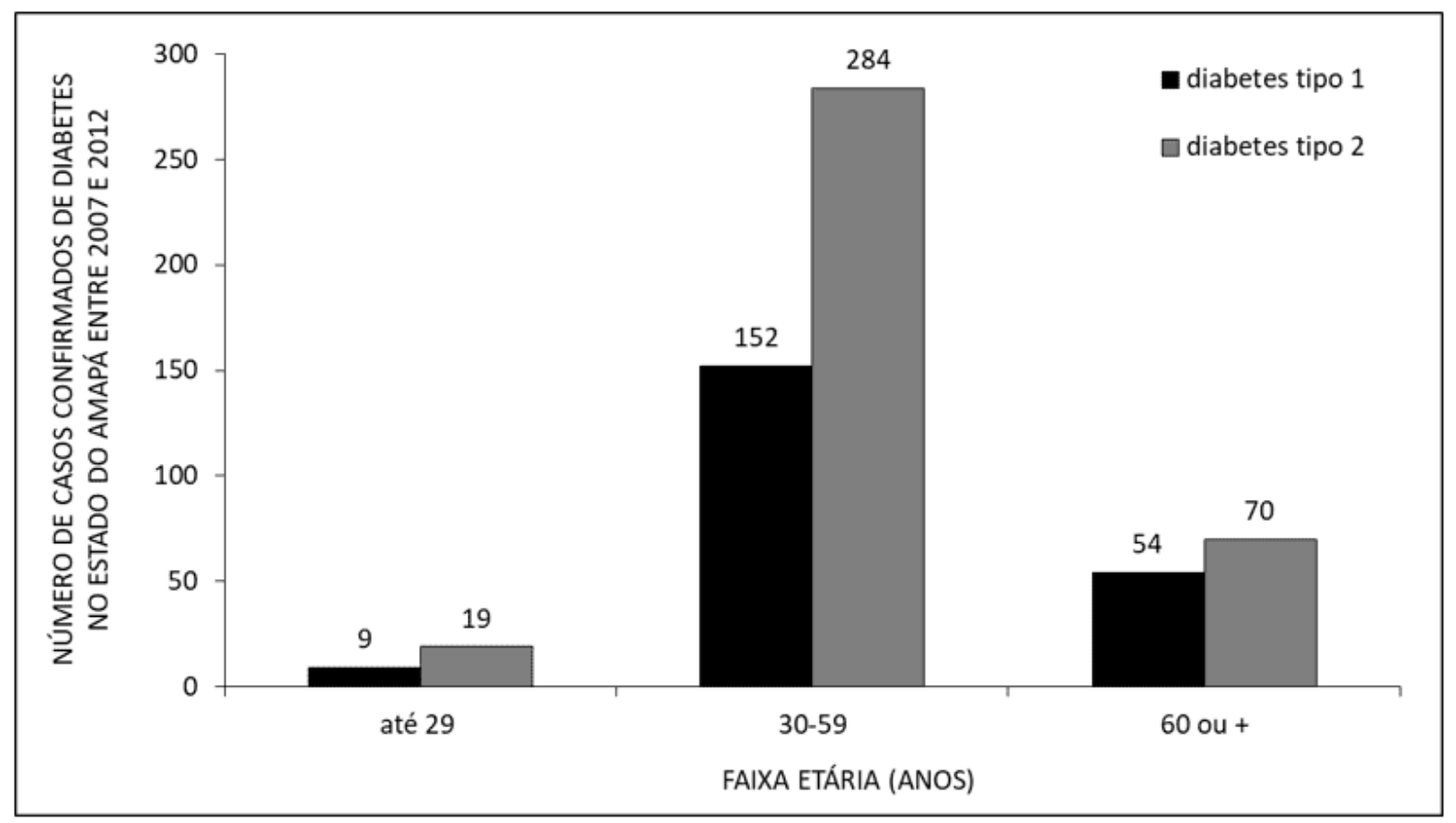

La figure 4 montre le nombre de cas confirmés de diabète de type 1 et 2 dans l'État d'Amapá entre 2007 et 2012 en fonction du poids. Le plus grand nombre de cas diagnostiqués de diabète de type 1 et 2 était celui des personnes sans surpoids.

RC: 66737

Disponible en: https://www.nucleodoconhecimento.com.br/sante/cas-de-diabete 
La figure 4 montre le nombre de cas confirmés de diabète de type 1 et 2 dans l'État d'Amapá entre 2007 et 2012 en fonction du poids.

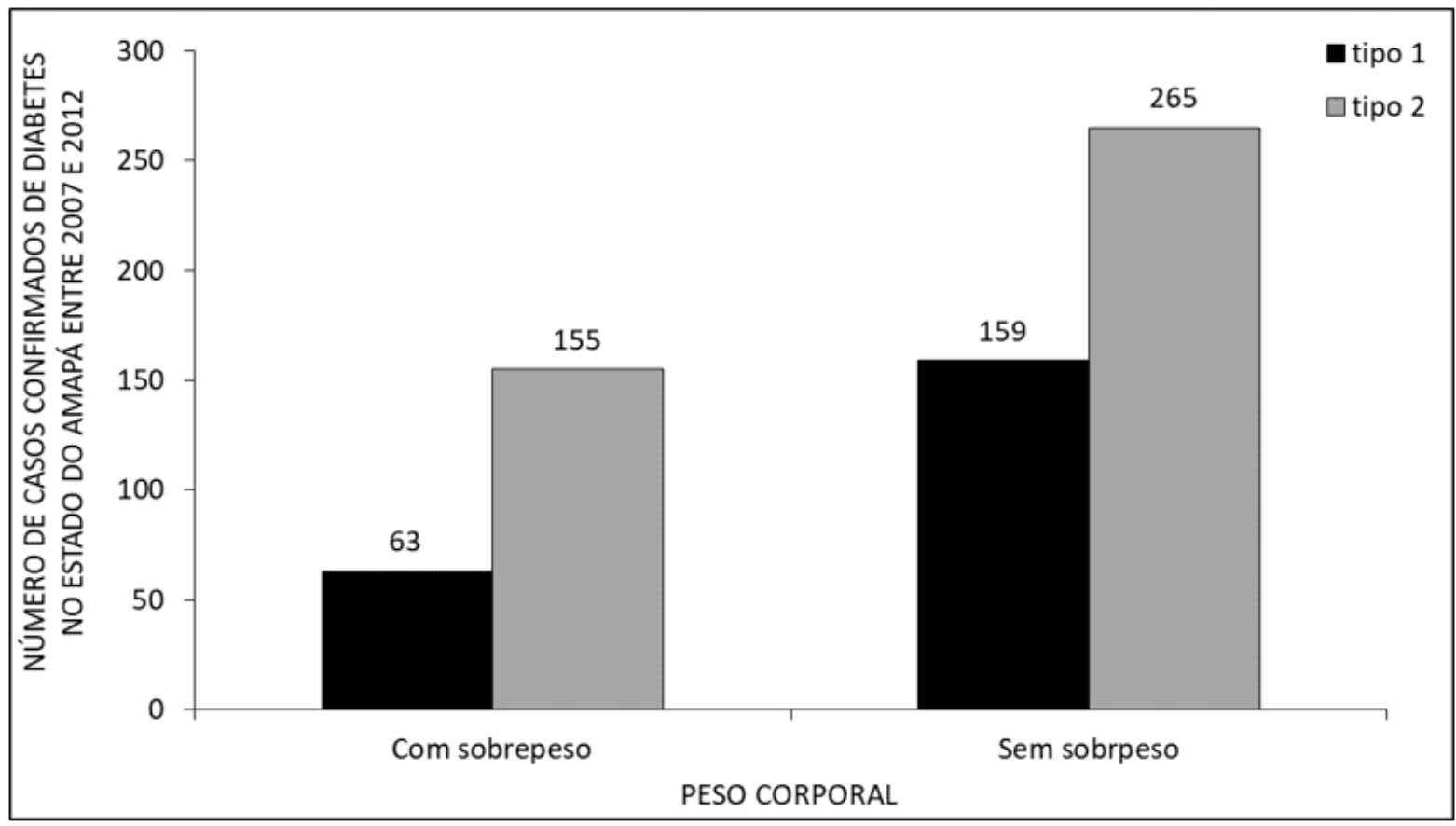

La figure 5 montre le nombre de cas confirmés de diabète de type 1 et 2 dans l'État d'Amapá entre 2007 et 2012 selon le mode de vie sédentaire. Le plus grand nombre de diagnostics de diabète de type 1 et 2 était celui des personnes non sédentaires.

RC: 66737

Disponible en: https://www.nucleodoconhecimento.com.br/sante/cas-de-diabete 
La figure 5 montre le nombre de cas confirmés de diabète de type 1 et 2 dans l'État d'Amapá entre 2007 et 2012 selon le mode de vie sédentaire.

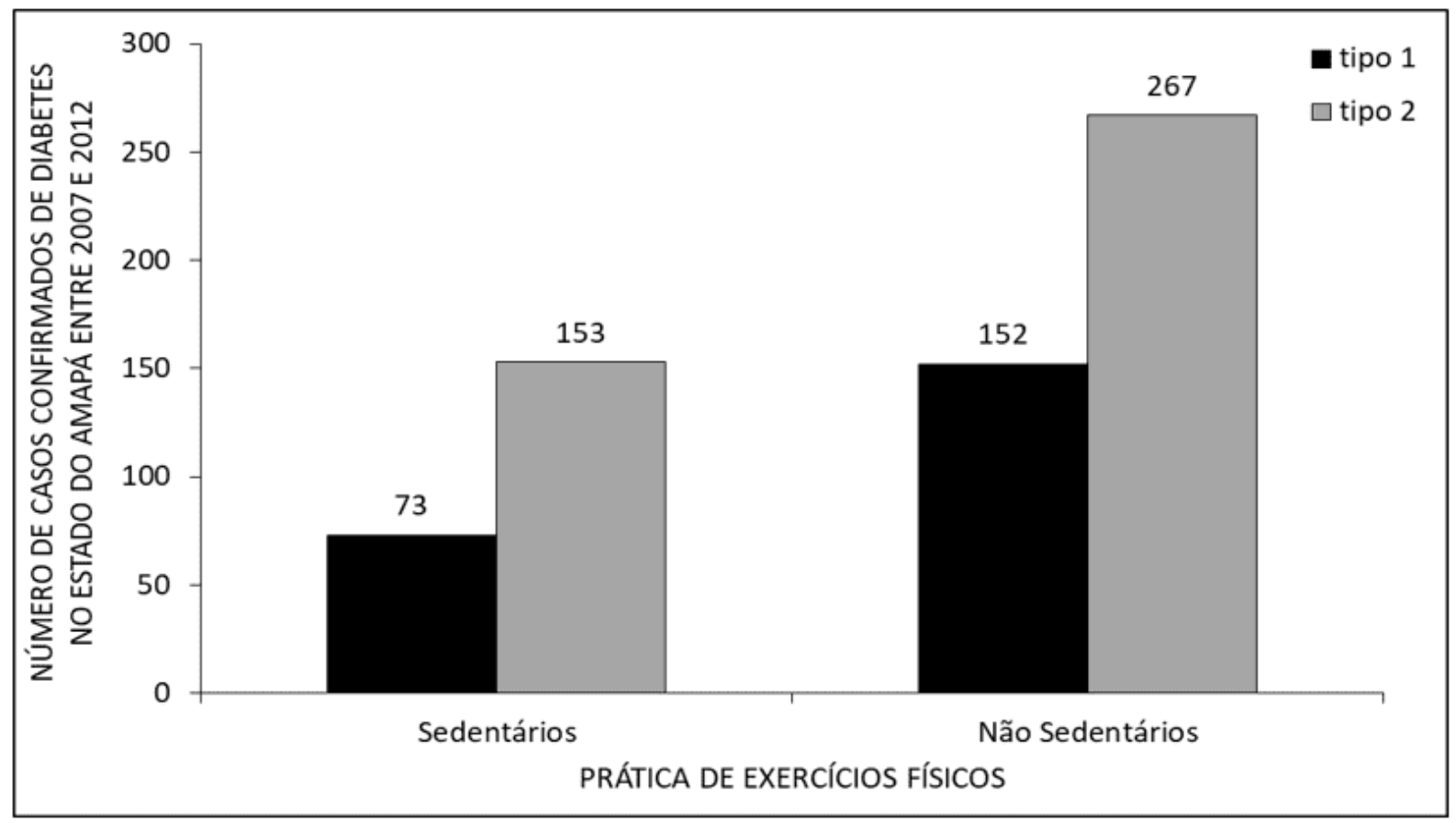

La figure 6 montre le nombre de cas confirmés de diabète de type 1 et 2 dans l'État d'Amapá entre 2007 et 2012 selon le tabagisme. Le plus grand nombre de diagnostics de diabète de type 1 et 2 était celui des non-fumeurs.

RC: 66737

Disponible en: https://www.nucleodoconhecimento.com.br/sante/cas-de-diabete 
La figure 6 montre le nombre de cas confirmés de diabète de type 1 et 2 dans l'État d'Amapá entre 2007 et 2012 selon le tabagisme.

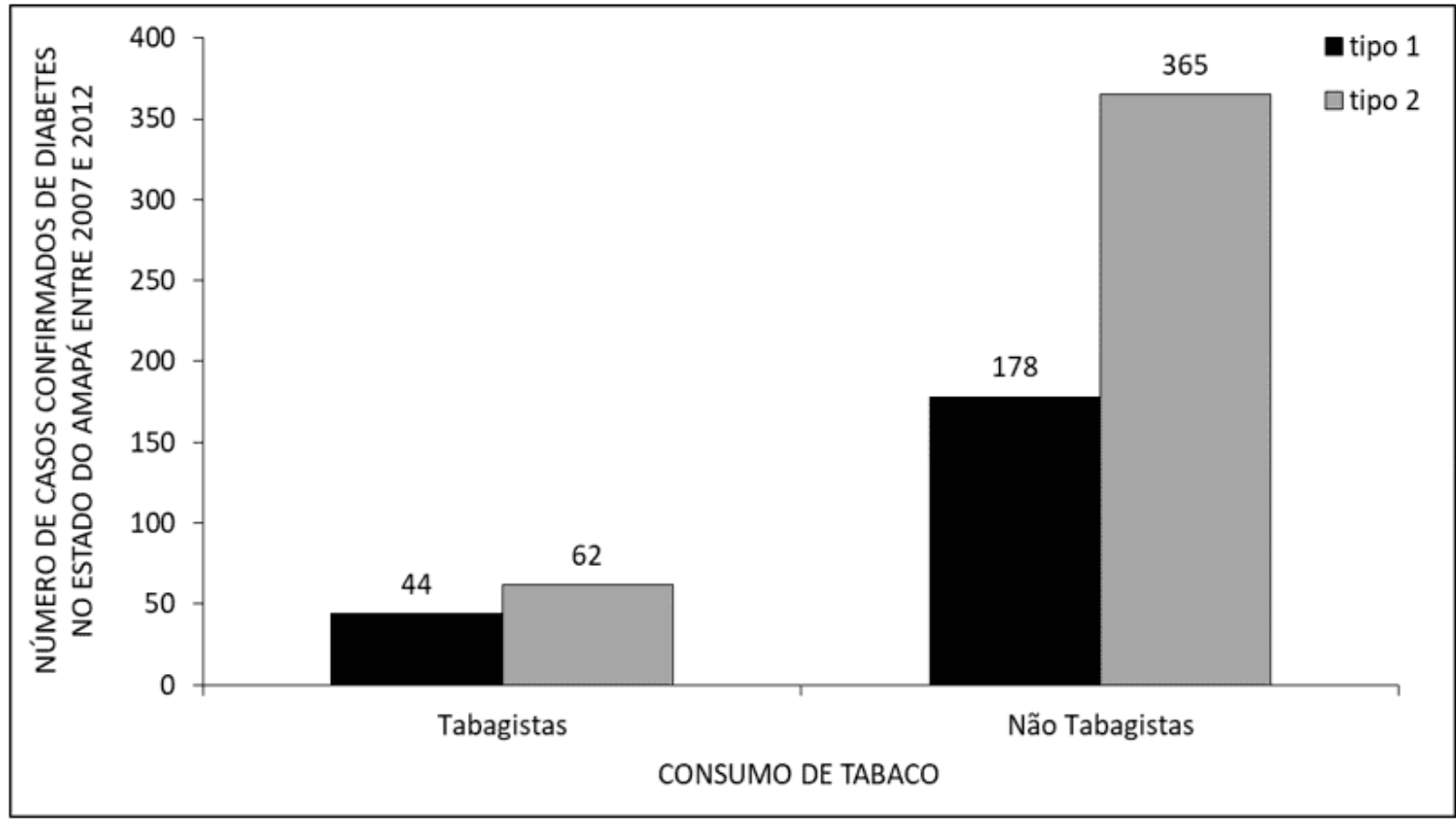

\section{DISCUSSION}

Le diabète sucré de type 2 (DM2) est l'une des principales maladies chroniques non transmissibles (MNT) et représente environ $90 \%$ de tous les cas actuels de diabète (COSTA et al., 2017). Ce facteur est très associé à la prévalence de l'obésité, car c'est l'un des principaux facteurs de risque pour le développement du DM2. Le nombre de patients obèses (qui ont DM2) qui reçoivent des soins spécifiques et bien structurés afin qu'ils puissent contrôler leur poids est très faible, et, par conséquent, il est noté que ce problème constitue un facteur de risque important pour la santé individuelle, parce que l'obésité représente un risque pour le développement de diverses maladies, telles que les maladies cardiovasculaires, influençant d'une manière largement négative le contrôle du DM2 (LIMA et al. , 2015).

Notez que la prévalence du diabète chez les femmes est élevée, et il y a plusieurs facteurs à analyser pour ce résultat. Dans une étude réalisée dans la ville de São

RC: 66737

Disponible en: https://www.nucleodoconhecimento.com.br/sante/cas-de-diabete 
Leopoldo, à Rio Grande do Sul, de nombreux paramètres et variables de ce problème ont été évalués. II a été noté que la prévalence la plus élevée de diabète s'est produite chez les femmes qui ont : âge entre 40 et 49 ans, état matrimonial marié, revenu inférieur à 1 salaire minimum, 1 à 3 enfants, tabagisme, hypertension artérielle systémique (HTA) et d'autres facteurs , comme l'obésité, qui est le facteur de risque le plus important de DM2 (DIAS-DA-COSTA et al., 2020).

Le diabète sucré de type 1 (DM1), également appelé diabète insulinodépendant, survient plus fréquemment chez les patients adolescents, et sa pathophysiologie implique la destruction de cellules bêta pancréatiques - responsables de la production d'insuline dans le corps - et, par conséquent, conduisant le corps à une carence en production d'insuline, rendant l'individu dépendant de l'utilisation de l'insuline synthétique. DM2 se produit habituellement après 30 ans, étant plus commun dans les individus entre 50 et 60 ans. (ABREU, 2017). Sa pathophysiologie est liée à la résistance à l'insuline et, par conséquent, l'action hypoglycémique effectuée par cette hormone ne se produit pas adéquatement, provoquant une augmentation de la production de glucose par le foie, contribuant largement à la quantité élevée d'insuline au niveau sanguin (BERTONHI et DIAS, 2018).

Bien qu'entre 2007 et 2012, le nombre de cas confirmés de DM1 et de DM2 à Amapá ait été plus élevé chez les personnes sans surpoids, non sédentaires et nonfumeurs, l'association entre le DM2 et ces conditions est bien connue. Une grande proportion de patients atteints de diabète sucré de type 2 sont obèses ou en surpoids. La pratique du tabagisme est responsable de l'augmentation exponentielle de la possibilité que l'individu développe un néoplasme, étant (isolément) la principale cause de cancer dans le monde entier. Le diabète a un lien intime avec le cancer, car il augmente la possibilité de développer des néoplasmes hépatiques, en plus des cancers du côlon, de l'endomètre, du sein et du pancréas. Ainsi, la prédisposition des fumeurs diabétiques aux néoplasmes est notée. En outre, le mode de vie sédentaire est caractérisé comme l'une des variables à considérer pour le développement de DM2. Dans une étude menée auprès d'étudiants de l'Université

RC: 66737

Disponible en: https://www.nucleodoconhecimento.com.br/sante/cas-de-diabete 
fédérale de Ceará (UFC), il a été noté le manque véhément d'exercice physique régulier, parfois justifié par le manque de motivation et de temps, en plus de la fatigue provenant du voyage quotidien de l'étudiant. L'absence de pratique quotidienne des exercices physiques peut favoriser l'embonpoint (surpoids et obésité), qui sont des facteurs de risque pour le développement du DM2 (LIMA et al., 2015; HOCAYEN et MALFATTI, 2010; LIMA et al., 2014).

\section{CONCLUSION}

Les diabètes sucrés de type 1 et 2 (DM1 et DM2) sont des maladies liées à des perturbations de la production ou à l'utilisation efficace de l'insuline. Le DM1 est une maladie dont la pathophysiologie n'est pas complètement connue et qui implique une prédisposition génétique, combinée à des facteurs environnementaux. DM2, d'autre part, même si elle a des bases génétiques, est très proche du mode de vie de l'individu, et l'obésité, l'embonpoint et le mode de vie sédentaire sont l'un des principaux facteurs de risque pour le développement de cette pathologie.

Il est à noter que, chez les diabétiques, la prévalence des femmes est très élevée. Des facteurs tels que l'âge entre 40 et 49 ans, le revenu inférieur à 1 salaire minimum, l'état matrimonial marié, 1 à 3 enfants, la pratique du tabagisme, l'hypertension artérielle systémique (HTA), et d'autres facteurs, tels que l'obésité sont responsables de ce problème.

DM1 se produit plus fréquemment dans les patients adolescents, et sa pathophysiologie implique la destruction des cellules bêta pancréatiques, causant dans le corps une insuffisance de production d'insuline, rendant l'individu dépendant de l'utilisation de l'insuline synthétique. Le DM2 survient plus fréquemment après 30 ans, étant plus fréquent chez les personnes âgées de 50 à 60 ans, et sa pathophysiologie est liée à la résistance à l'insuline et, compte tenu de cela, l'action hypoglycémique effectuée par l'insuline ne se produit pas adéquatement dans le corps, causant, comme la chronicité de la maladie se produit, une série de

RC: 66737

Disponible en: https://www.nucleodoconhecimento.com.br/sante/cas-de-diabete 
conséquences négatives pour les organismes, avec des lésions macro et microvasculaires et entre autres.

Le tabagisme, ainsi que le mode de vie sédentaire et l'embonpoint sont des facteurs de risque importants pour le développement du DM2. Le diabète sucré de type 2 fournit le développement de diverses lésions nerveuses organiques. En outre, le DM2, par sa chronicité, permet le développement des rétinopathies, des néphropathies et d'autres conditions négatives à la santé de l'individu.

\section{RÉFÉRENCES}

ABREU, L. C. S. Diabetes na Terceira Idade. Revista Científica Multidisciplinar Núcleo do Conhecimento, v. 2, p. 111-131, 2017.

ADA. American Diabetes Association; Diagnosis and Classification of Diabetes Mellitus; Diabetes Care, Volume 37, Supplement 1, January 2014.

BERTONHI, L. G.; DIAS, J. C. R. Diabetes mellitus tipo 2: aspectos clínicos, tratamento e conduta dietoterápica. Revista Ciências Nutricionais Online, v. 2, n. 2, p. 1-10, 2018.

BRASIL. Ministério da Saúde. Secretaria de Atenção à Saúde. Departamento de Atenção Básica. Diabetes Mellitus / Ministério da Saúde, Secretaria de Atenção à Saúde, Departamento de Atenção Básica. - Brasília : Ministério da Saúde, 2006.

BRASIL. Portal Brasil. Diabetes traz consequências graves se não for controlado. 2012. disponível em: <http://www.brasil.gov.br/saude/2012/04/diabetes>. Acessado Em: 19/09/2017.

CORTEZ. D.N; Reis, I.A; Souza, D.A.S; Macedo, M.M.L; Torres H.C Complicações e o tempo de diagnóstico do diabetes mellitus na atenção primária Acta Paulista de Enfermagem, vol. 28, núm. 3, 2015, pp. 250-255.

$\mathrm{RC}: 66737$

Disponible en: https://www.nucleodoconhecimento.com.br/sante/cas-de-diabete 
COSTA, A. F.; FLOR, L. S.; CAMPOS, M. R.; OLIVEIRA, A. F.; COSTA, M. F. S.; SILVA, R. S.; LOBATO, L. C. P.; SCHRAMM, J. M. A. Carga do diabetes mellitus tipo 2 no Brasil. Cad. Saúde Pública, v. 33, n. 2, p. 1-14, 2017.

DIAS-DA-COSTA, J. S.; SILOCCHI, C.; SCHWENDLER, S. C.; MORIMOTO, T.; MOTTIN, V. H. M.; PANIZ, V. M. V.; BAIRROS, F. S.; OLINTO, M. T. A. Prevalência de diabetes mellitus autorreferido em mulheres e fatores associados: estudo de base populacional em São Leopoldo, Rio Grande do Sul, 2015. Epidemiol. Serv. Saude, v. 29, n. 2, p. 1-12, 2020.

HOCAYEN, P. A. S.; MALFATTI, C. R. M. Tabagismo em pacientes diabéticos: predisposição às doenças crônico-degenerativas e neoplasia. Cinergis, v. 11, n. 2, p. 19-25, 2010.

LIMA, A. C. S.; ARAÚJO, M. F. M.; FREITAS, R. W. J. F.; ZANETTI, M. L.; ALMEIDA, P. C.; DAMASCENO, M. M. C. Fatores de risco para diabetes mellitus tipo 2 em universitários: associação com variáveis sociodemográficas. Rev. LatinoAm. Enfermagem, v. 22, n. 3, p. 484-490, 2014.

LIMA, L. L.; SÁ, A. D.; FIGUEIREDO, A. S.; MUÑOZ, R. L. S. Prevalência de sobrepeso e obesidade em diabéticos tipo 2 atendidos no ambulatório de Endocrinologia de um Hospital Universitário. Rev Soc Bras Clin Med, v. 13, n. 4, p. 251-256, 2015.

MORA, G.P.C.; Abascal I.C.; Sanabria, G. Sobrepeso, obesidad y diabetes mellitus 2 en adolescentes de América Latina en 2000-2010;Revista Cubana de Medicina General Integral. v. 31, n. 3, 217-231, 2015.

SANTOS, M. S, Freitas, N. M; Pinto, F. O; O DIABETES MELLITUS TIPO 1 E TIPO 2 E SUA EVOLUÇÃO NO MUNICÍPIO DE QUISSAMÃ-RJ; Revista Científica Interdisciplinar, vol 1, No 1, 2014.

RC: 66737

Disponible en: https://www.nucleodoconhecimento.com.br/sante/cas-de-diabete 
UFRGS. RegulaSUS. Diabetes Mellitus. Disponível em: $<$ https://www.ufrgs.br/telessauders/documentos/protocolos_resumos/endocrino_resu mo_diabetes_TSRS_20160324.pdf >.Acesso em: 19/09/2017.

Soumis : Décembre 2020.

Approuvé : Décembre 2020.

RC: 66737

Disponible en: https://www.nucleodoconhecimento.com.br/sante/cas-de-diabete 